\title{
Switching to an alternative biological agent in juvenile idiopathic arthritis (II)
}

\author{
S Murias, A Remesal, L Latorre, M Gomez, R Merino \\ From 18th Pediatric Rheumatology European Society (PReS) Congress \\ Bruges, Belgium. 14-18 September 2011
}

\section{Background}

Juvenile idiopathic arthritis (JIA) is a heterogeneous disease, and it's associated with an increased use of various biological agents in recent years.

\section{Aim}

Evaluate clinical response to current biological agent in JIA patients.

\section{Methods}

This is a retrospective study of 109 JIA patients from a tertiary centre (see poster 1 ). Variables included were: current biological treatment (both the first agent used as the second, third or fourth when it had been switched) and duration of treatment (years with a new agent or since reintroduction of an agent used before) plus physician visual analogue scale (ph-VAS), erythrocyte sedimentation rate (ESR) and C-reactive protein (CRP) at baseline and at the end of the study. Concomitant therapy with methotrexate (MTX) was also recorded.

\section{Results}

Duration of treatment with the current biological agent was $1.2 \pm 1(0.1-4.9)$ median 0.8 years. This agent was etanercept (ETA) in 58 cases, adalimumab (ADA) in 16, tocilizumab (TCZ) in 11, anakinra (AK) in 9 and infliximab (IFX) in 2. Thirteen patients were off treatment with biological agents, 12 due to inactive disease and one because of inefficacy.

Concomitant treatment with MTX was observed in 8/ 58 (14\%) patients with ETA, 4/16 (25\%) with ADA, 5/11 (46\%) with TCZ, $1 / 2$ (50\%) with IFX and none with AK. Table 1.

\section{Conclusion}

The results indicate that reintroduction of a previously effective biological agent may be successful; besides, switching to a second, third or even fourth biological agent in those patients whose disease remains active, can be effective as well.

Published: 14 September 2011

doi:10.1186/1546-0096-9-S1-P163

Cite this article as: Murias et al:: Switching to an alternative biological agent in juvenile idiopathic arthritis (II). Pediatric Rheumatology 20119 (Suppl 1):P163.

Table 1 Analytical and clinical response to current biological agent

\begin{tabular}{|c|c|c|c|c|c|c|c|c|c|c|c|c|}
\hline & Ph-VAS & & & & ESR & & & & CRP & & & \\
\hline & Initial* & End* & 0 & $p$ & Initial* & End* & 0 & $p$ & Initial* & End* & 0 & $p$ \\
\hline ETA & 3.25 & 0.5 & -6 & 0.00 & 28 & 12 & -5 & 0.00 & 13.2 & 2.3 & -5 & 0.00 \\
\hline ADA & 2.42 & 0.4 & -3 & 0.01 & 17 & 13 & -1 & 0.26 & 5.58 & 1.2 & -2 & 0.06 \\
\hline$A K$ & 5.36 & 0.00 & -3 & 0.01 & 62 & 9 & -2 & 0.02 & 69.5 & 2.9 & -2 & 0.03 \\
\hline $\mathrm{TCZ}$ & 6.45 & 0.86 & -3 & 0.01 & 46 & 4 & -3 & 0.01 & 95.6 & 0.6 & -3 & 0.01 \\
\hline IFX & 4.9 & 0.00 & & & 47 & 6 & & & 32.8 & 0.9 & & \\
\hline
\end{tabular}

*Values are expressed as mean.

\section{() Biomed Central}

